\begin{tabular}{|c|l|}
\hline Title & $\begin{array}{l}\text { Vanishing fine structure splittings in telecommunication-wavelength quantum dots grown on (111)A surfaces by } \\
\text { droplet epitaxy }\end{array}$ \\
\hline Author(s) & $\begin{array}{l}\text { Liu, Xiangming; Ha, Neul; Nakajima, Hideaki; Mano, Takaaki; Kuroda, Takashi; Urbaszek, Bernhard; Kumano, } \\
\text { Hidekazu; Suemune, Ikuo; Sakuma, Y oshiki; Sakoda, Kazuaki }\end{array}$ \\
\hline Citation & $\begin{array}{l}\text { Physical Review B, 90(8), 081301-1-081301-6 } \\
\text { https://doi.org/10.1103/PhysRevB.90.081301 }\end{array}$ \\
\hline Issue Date & 201408-27 \\
\hline Doc URL & http://hdl.handle.net/2115/59633 \\
\hline Rights & O2014 A merican Physical Society \\
\hline Type & article \\
\hline File Information & Liu_PRB2015.pdf \\
\hline
\end{tabular}

Instructions for use 


\title{
Vanishing fine-structure splittings in telecommunication-wavelength quantum dots grown on (111)A surfaces by droplet epitaxy
}

\author{
Xiangming Liu, ${ }^{1}$ Neul Ha, ${ }^{1,2}$ Hideaki Nakajima, ${ }^{3}$ Takaaki Mano, ${ }^{1}$ Takashi Kuroda,,${ }^{1,2, *}$ Bernhard Urbaszek, ${ }^{4}$ \\ Hidekazu Kumano, ${ }^{3}$ Ikuo Suemune, ${ }^{3}$ Yoshiki Sakuma, ${ }^{1}$ and Kazuaki Sakoda ${ }^{1}$ \\ ${ }^{1}$ National Institute for Materials Science, 1-1 Namiki, Tsukuba 305-0044, Japan \\ ${ }^{2}$ Graduate School of Engineering, Kyushu University, NIMS, Tsukuba 305-0044, Japan \\ ${ }^{3}$ Research Institute for Electronic Science, Hokkaido University, Sapporo 001-0021, Japan \\ ${ }^{4}$ Université de Toulouse, INSA-CNRS-UPS, LPCNO, 135 Av. Rangueil, 31077 Toulouse, France \\ (Received 17 June 2014; revised manuscript received 11 August 2014; published 27 August 2014)
}

\begin{abstract}
The emission cascade of a single quantum dot is a promising source of entangled photons. A prerequisite for this source is the use of a symmetric dot analogous to an atom in a vacuum, but the simultaneous achievement of structural symmetry and emission in a telecom band poses a challenge. Here we report the growth and characterization of highly symmetric InAs/InAlAs quantum dots self-assembled on $C_{3 v}$ symmetric InP(111)A. The broad emission spectra cover the $\mathrm{O}(\lambda \sim 1.3 \mu \mathrm{m}), \mathrm{C}(\lambda \sim 1.55 \mu \mathrm{m})$, and $L(\lambda \sim 1.6 \mu \mathrm{m})$ telecom bands. The distribution of the fine-structure splittings is considerably smaller than those reported in previous works on dots at similar wavelengths. The presence of dots with degenerate exciton lines is further confirmed by the optical orientation technique. Thus, our dot systems are expected to serve as efficient entangled photon emitters for long-distance fiber-based quantum key distribution.
\end{abstract}

DOI: 10.1103/PhysRevB.90.081301

Semiconductor quantum dots (QDs) are expected to play a central role in quantum information networks. A noteworthy device based on dots is the solid-state single photon source, which ensures absolute security in quantum key distribution (QKD) [1]. Since QDs can confine charged carriers in nanometer-sized regions, recombination enables single photons to appear on demand, i.e., synchronously with a master clock shared in networks [2]. QKD over a $50 \mathrm{~km}$ commercial fiber has already been demonstrated with QD photon sources, which emitted at a wavelength of $1.5 \mu \mathrm{m}$ [3]. The transmission distance in that work was limited purely by the absorption loss of silica fibers. Exceeding this fundamental limit requires the development of quantum link protocols, which exploit the nonlocality inherent in quantum theory. An efficient source of entangled photon pairs is a key element in the realization of such protocols, examples of which include quantum teleportation [4] and entanglement swapping [5].

The generation of entangled photons with semiconductor QDs is directly linked to the singlet configuration of two excitons $(X)$, which form a biexciton $(X X)$. Eventually, two photons associated with the $X X-X$ cascade show polarization correlations independent of the choice of measurement basis, yielding quantum entanglement in the polarization state [6,7]. However, commonly studied QDs exhibit considerable finestructure splittings (FSS) [8-12], which exclude entanglement in emitted photons [13]. Numerous attempts have been made to suppress FSS and recover the symmetry of QDs grown on conventional (001) oriented substrates [14-21]. An average FSS of $\sim 4 \mu \mathrm{eV}$ was also measured in GaAs QDs on nanohole template (001) substrates [22]. However, from a practical point of view, the reproducible and self-assempled growth of symmetric dots with (at least) near-zero FSS is highly desirable.

*kuroda.takashi@nims.go.jp
PACS number(s): 78.67.Hc, 73.21.La, 81.07.Ta

A noteworthy strategy for achieving such high QD symmetry is the application of $C_{3 v}$ symmetric (111) surfaces to the growth substrate, as was predicted theoretically [23]. Although QD growth in the Stranski-Krastanow (SK) mode is prohibited along the [111] axis, the use of patterned substrates [24] and droplet epitaxy [25,26] makes it possible to grow QDs on (111) substrates. Hence, a great reduction in FSS was observed in these QDs [26,27], which led to the demonstration of entangled photon emission in pyramidal QDs on patterned (111)B substrates [28], and the filtering-free violation of Bell's inequality for droplet epitaxial GaAs/AlGaAs dots on GaAs(111)A [29]. Note that all these studies dealt with visible wavelength photons. Material challenges have meant that the development of QD sources in telecom bands has achieved less progress. The simultaneous realization of small FSS and telecom-band emission is a great challenge.

In this work, we report on the growth and characterization of telecom-band InAs quantum dots on (111)A substrates. For this purpose, we focus on the use of droplet epitaxy, which is not strain driven, thereby enabling us to choose a variety of materials and surface orientations. Though most previous works on droplet epitaxy dealt with lattice-matched systems, the versatility of this technique makes it possible to create QDs on lattice-mismatched systems targeting telecom-band emission. The successful growth of InAs/InAlAs QDs on $\operatorname{InP}(111) \mathrm{A}$ has recently been demonstrated [30]. Here we use newly created QDs with a lower surface density, which allows a systematic study of FSS and the symmetry characteristics of dots. The measured FSS reveals an average value of $25 \mu \mathrm{eV}$, which is considerably smaller than those in previous studies on SK-grown dots at similar wavelengths [31-33]. Moreover, the presence of QDs with nearly perfect exciton degeneracy is confirmed using the optical orientation technique. Thus our source is expected to serve as a promising candidate for highly efficient entangled photon sources, which do not require the use of serious temporal gating to improve the degree of quantum correlation [34]. 

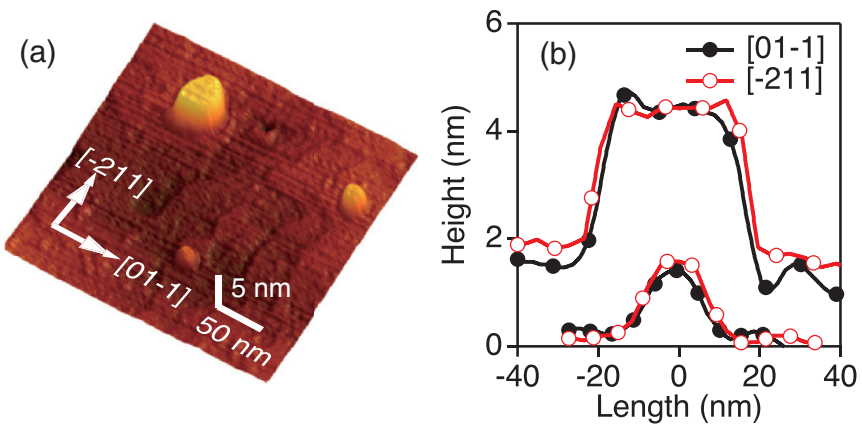

FIG. 1. (Color online) (a) AFM image of the sample surface of InAs dots on $\operatorname{In}_{0.52} \mathrm{Al}_{0.48} \mathrm{As} / \mathrm{InP}(111) \mathrm{A}$. (b) Cross sections along $\left[\begin{array}{ll}0 & 1-1]\end{array}\right]$ and $[-211]$ for a relatively large dot (top) and a small dot (bottom)

The sample investigated in this study was grown by droplet epitaxy using a conventional molecular beam epitaxy apparatus $[35,36]$. After growing a 150 -nm-thick $\operatorname{In}_{0.52} \mathrm{Al}_{0.48} \mathrm{As}$ barrier layer on an $\operatorname{InP}(111) \mathrm{A}$ substrate at $470^{\circ} \mathrm{C}$, we cooled the substrate to $320^{\circ} \mathrm{C}$ and supplied 0.4 monolayers of indium, which led to the formation of indium droplets. Next, we supplied an $\mathrm{As}_{4}$ flux of $3 \times 10^{-5}$ Torr to crystallize the indium droplets into InAs dots at $270^{\circ} \mathrm{C}$. The sample was then annealed at $370{ }^{\circ} \mathrm{C}$ and capped with another $\operatorname{In}_{0.52} \mathrm{Al}_{0.48} \mathrm{As}$ barrier layer with a thickness of $75 \mathrm{~nm}$.

For atomic force microscopy (AFM) analysis, an additional QD layer was grown on the top of the sample. Figure 1(a) shows a three-dimensional view of the surface, which reveals the presence of disklike dots with $3.0( \pm 1.0) \mathrm{nm}$ in height and $38( \pm 10) \mathrm{nm}$ in diameter. The dot density is as low as $3.2 \times$ $10^{9} \mathrm{~cm}^{-2}$, which makes it possible to isolate single dots using conventional microphotoluminescence techniques. Figure 1(b) shows the AFM cross sections for two example QDs. Cross sections obtained along the orthogonal in-plane directions, $[01-1]$ and [ -211$]$, are almost identical, which supports the high lateral symmetry in the dot shape without any elongations. This symmetric characteristic is a consequence of QD growth on (111) substrates.

For the photoluminescence (PL) measurement we used a continuous wave laser emitting at a wavelength of $705 \mathrm{~nm}$ for excitation above the barrier band gap. The laser light was focused on the sample using a near-infrared microscope objective with a numerical aperture of 0.65 . To reduce the spot size, a hemispherical solid immersion lens with a refractive index of two was positioned on the sample. In this setup, approximately 20 dots are in the excitation spot (diameter $\sim 0.9 \mu \mathrm{m}$ ). Nevertheless, we can isolate a single dot in spectral domain from a dot ensemble with broad size distribution. Spontaneously emitted photons were collected with the same objective, and then fed into a $50-\mathrm{cm}$ focal length polychromator equipped with an InGaAs array detector. The spectrometer had a resolution of $55 \mu \mathrm{eV}(0.08 \mathrm{~nm})$ with a full width at half maximum (FWHM) at a wavelength of $1.3 \mu \mathrm{m}$. The linearly polarized PL spectra were recorded as a function of the polarization angle. With a Gaussian fit to the emission lines, we were able to determine the spectral peak shift (and the absolute value of FSS) with a resolution as high as $4 \mu \mathrm{eV}$. All the experiments were performed at $10 \mathrm{~K}$.
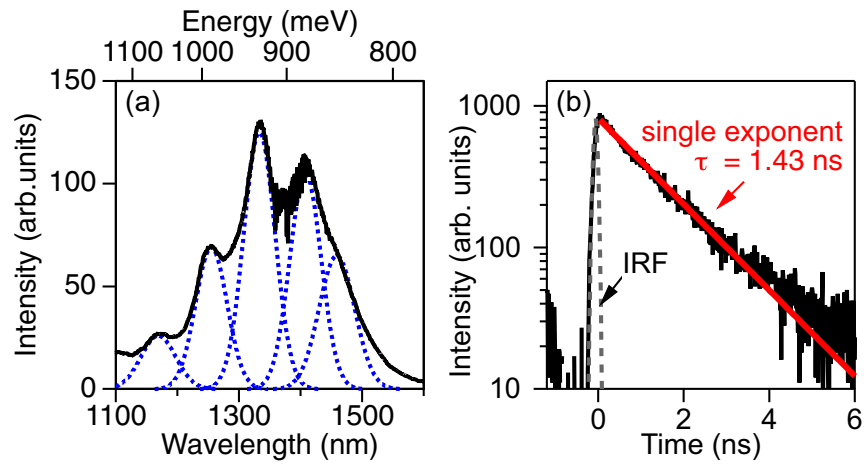

FIG. 2. (Color online) (a) PL spectrum of the ensemble of dots. The blue broken lines show the results of multiple-peak fit with assuming Gaussian broadening for each peak. (c) Time-resolved PL signals after picosecond pulsed excitation. The gray broken line shows the instrumental response function (IRF). The red line shows a single exponential fit to the decay data with a lifetime of $1.43 \mathrm{~ns}$.

Figure 2(a) shows the low-temperature PL spectrum of the dot ensembles. The PL spectrum spreads in a 1.1-1.6 $\mu \mathrm{m}$ wavelength range, which covers the $\mathrm{O}(\lambda \sim 1310 \mathrm{~nm}), \mathrm{C}$ $(\lambda \sim 1550 \mathrm{~nm})$, and $L(\lambda \sim 1600 \mathrm{~nm})$ telecom bands. The spectrum consists of several split peaks, among which highyield emissions are centered at $\sim 930 \mathrm{meV}(1333 \mathrm{~nm})$ with an FWHM of $40 \mathrm{meV}$. The appearance of multiple peaks can be attributed to the different families of QDs with heights varying in monolayer steps [26]. Note that we measured the PL at a sufficiently low power where contribution from excited states can be excluded. The AFM analysis suggests that the QDs have a flat shape with a height that ranges from 2 to 6 monolayers, which is consistent with the observed spectral profile.

Figure 2(b) shows the PL decay signals of the dot ensembles at wavelengths around $1.3 \mu \mathrm{m}$ after pulsed excitation. For this measurement, we used a mode-locked Ti:sapphire laser for excitation $(\lambda \sim 785 \mathrm{~nm})$ and a superconducting single photon detector (SSPD) for detection. The decay curve reveals a single exponent with a decay time of $1.43 \mathrm{~ns}$, which agrees with the theoretical decay time of spontaneous emission on the assumption that the QDs have the same transition strength as the bulk value. The similar decay times have been confirmed in telecom-wavelength QDs grown for different substrate orientations [37]. Thus, the observed PL decay is likely governed by intrinsic carrier recombination and free of any nonradiative process, as a consequence of the high crystalline quality of dots. The homogeneous linewidth, which gives the maximum limit of FSS for entangled photon emission, is thus $\sim 0.5 \mu \mathrm{eV}$ for our dots.

Figure 3(a) shows the typical PL spectrum of a single dot. Four emission lines are observed, and assigned, from the high-energy side, as $X, X^{+}, X X$, and $X^{-}$, where $X^{+(-)}$ refers to positively (negatively) charged excitons. These assignments are based on the measurement of the excitation power dependence of the emission lines [Fig. 3(b)], where almost linear and quadratic behaviors were observed for $X$ and $X X$, respectively. The assignment of $X^{+}$and $X^{-}$is further supported by an optical orientation measurement, as described later. 

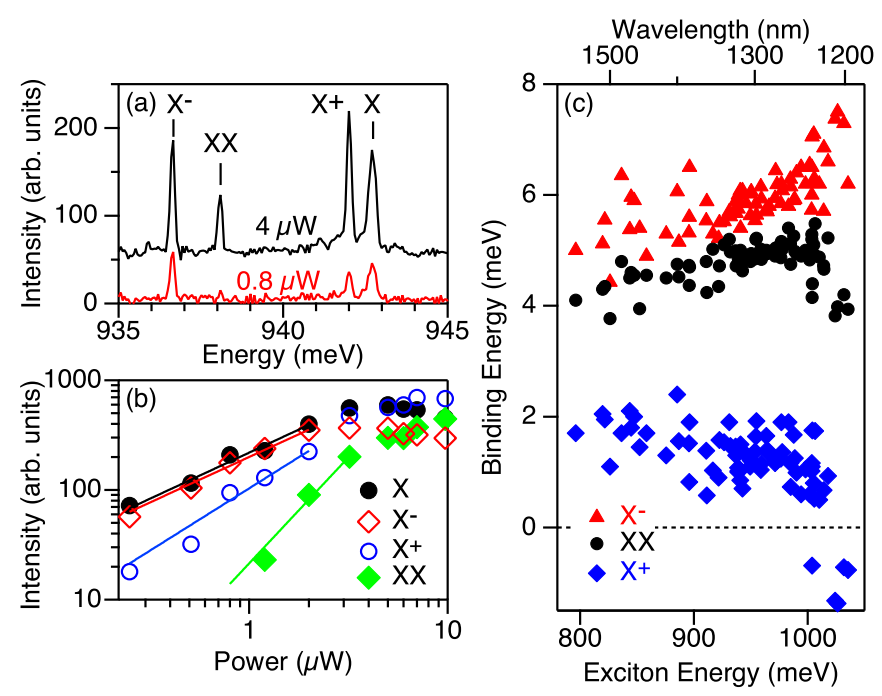

FIG. 3. (Color online) (a) Typical PL spectra of a single QD with an excitation power of $4 \mu \mathrm{W}$ and $0.8 \mu \mathrm{W}$. (b) PL intensity as a function of excitation power for each exciton complex. The solid lines show power lows, $I \propto P^{\alpha}$, where $\alpha=0.8,0.8,1.1$, and 1.8 for $X, X^{-}, X^{+}$, and $X X$, respectively. (c) Evolution of the binding energy with the $X$ energy for $X^{-}$(red triangles), $X X$ (black circles), and $X^{+}$(blue diamonds).

Figure 3(c) shows the binding energy of each exciton complex as a function of $X$ energy. Here the binding energy is defined as the energy difference between $X$ and the exciton complex. It reveals that, with increasing $X$ energy, the binding energy of $X^{-}$increases, that of $X^{+}$decreases, and that of $X X$ has intermediate values. The mirror symmetric evolution of $X^{-}$and $X^{+}$is induced by the mean-field contribution to exciton charging [38]. The positive binding energy of $X^{+}$, which is not common for SK dots, but frequently observed for droplet dots, implies the impact of nonseparable correlation to multiparticle binding [38]. Note that the spectral profile of exciton complexes is known to show a sensitive dependence on dot structure [39]. Therefore, the observation of a clear and less dispersive evolution in the binding energy suggests that the shape and other microscopic parameters of dots with a given size are almost identical. This is likely to be due to the kinetically limited formation of dots for droplet epitaxy. Consequently, dots on (111) substrates become rather symmetric as microscopic randomness is effectively suppressed.

Figures 4(a) and 4(b) show the evolution of the $X$ peak energy when the linear polarization axis is rotated for two dots. The precise quantification of FSS is based on sinusoidal fitting to these evolutions, where the magnitude (absolute value) of FSS is defined as the amplitude of sine curves, and the polarization axis $\phi$ is defined as the first maximum phase. Thus, the angle of $\phi$ corresponds to the polarization axis of the high-energy $X$ line among two split lines with orthogonal polarization. As shown by Figs. 4(a) and 4(b), both the FSS magnitude and polarization axis differ dot by dot.

The statistical results for FSS over $\sim 50$ dots are summarized in Fig. 4(c), where the FSS magnitude is plotted as a function of $X$ energy. A histogram of the FSS values is also shown in the Supplemental Material [40]. The FSS ranges

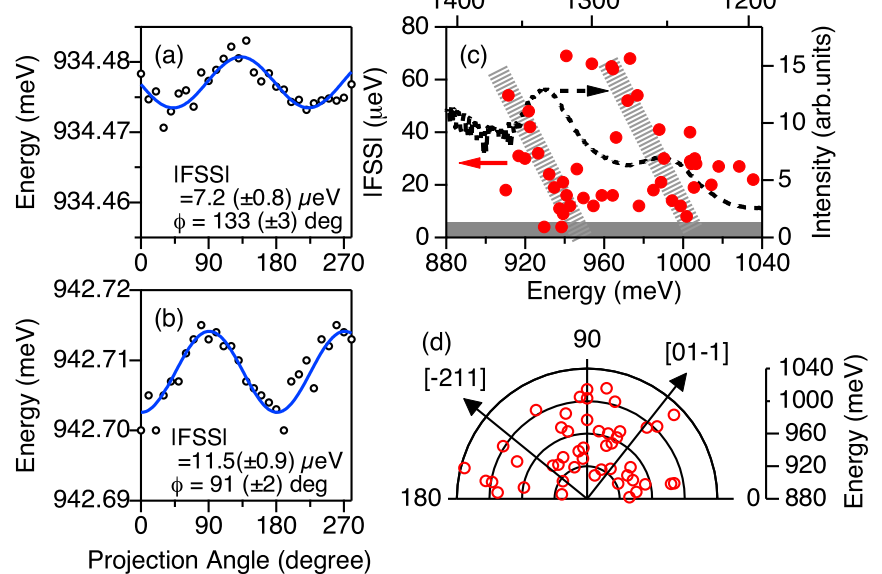

FIG. 4. (Color online) (a), (b) Energy shifts of the $X$ line with rotating the axis of linear polarization for two dots. The blue lines are sinusoidal fits to the data. (c) Absolute value of FSS measured on single QDs (red circles). The broken line shows the ensemble PL spectrum. The shaded thick lines are guides to the eye. (d) Polarization axis with respect to the emission energy. Two orthogonal in-plane axes [0 $1-1]$ and $\left[\begin{array}{lll}-2 & 1 & 1\end{array}\right]$ are shown by arrows. The equivalent directions appear with every $120^{\circ}$ rotation due to the $C_{3 v}$ point-group symmetry.

between 70 and $3 \mu \mathrm{eV}$, where the minimum value is smaller than the error width of the present analysis $(4 \mu \mathrm{eV}$, shown by the shaded region). The FSS average value is $25 \mu \mathrm{eV}$, which is considerably smaller than those of SK-grown QDs in the telecom band [31-33]. Note that two families of QDs with different monolayer heights are present in Fig. 4(c), as shown by the two peaks in the ensemble spectrum (broken line). Each family with a given height reveals a trend where the FSS decreases as the energy increases. This implies that high-energy QDs have a smaller in-plane size and higher lateral symmetry. The impact of the lateral-size reduction on FSS minimization was also confirmed in a previous study on FSS control by high-temperature annealing [14].

Figure 4(d) shows the direction of the polarization axis with respect to the $X$ energy. They are randomly distributed, without showing significant correlations with the in-plane crystallographic axes. The absence of a preferential direction in the (111) plane suggests a high probability of finding QDs with negligible FSS over a broad spectral range.

The presence of dots with effectively zero FSS can be confirmed by measuring circularly polarized emission signals. In the presence of a finite FSS value, the polarization state of the emission light is expected to oscillate temporally between the left- and right-handed circular polarizations, where the oscillation period is determined by the inverse of the FSS. Therefore, in time-integrated experiments we cannot observe a high degree of circular polarization. By contrast, in the absence of FSS, circular polarization remains in time-integrated signals. Thus, the observation of circular polarization provides a sufficient condition for exciton degeneracy. The measurement principle is analogous to the well-known Hanle measurement, and was used to monitor FSS cancellation by an electric field [41]. Note that we use the $1.76-\mathrm{eV}$ excitation light, which is 

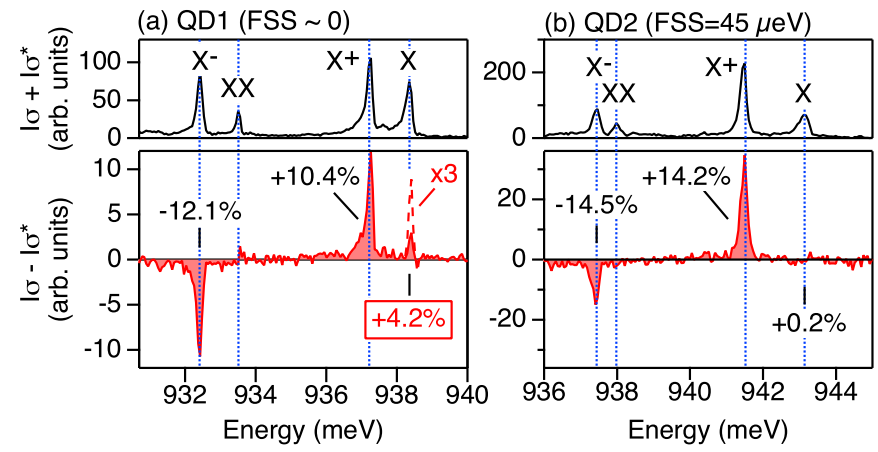

FIG. 5. (Color online) Circularly polarized emission signals with a nonresonant circularly polarized pump at a wavelength of $705 \mathrm{~nm}$ for (a) a QD without a detectable FSS, and (b) a QD with a finite value of FSS. Upper panels show unpolarized spectra. Lower panels show differential spectra between cocircular signals and cross-circular signals. The degree of circular polarization, $\left(I_{\sigma}-I_{\sigma^{*}}\right) /\left(I_{\sigma}+I_{\sigma^{*}}\right)$, of each emission line is also indicated. The acquisition time was $100 \mathrm{~s}$ for (a) and $20 \mathrm{~s}$ for (b).

predominantly absorbed by the unstrained InAlAs barrier with a direct band gap at $1.52 \mathrm{eV}$. Thus, the circularly polarized light induces spin-polarized electron injection from the heavy- and light-hole bands, but not from the split-off band. The maximum degree of spin polarization is therefore limited by 0.5 based on the assumption of fast hole relaxation. To avoid the effect of dynamic nuclear polarization [42], we set the excitation power at a sufficiently low level, where the average exciton population in the dot was $\sim 0.5$.

Figures 5(a) and 5(b) show optical orientation results for a selected dot without a detectable FSS $(<4 \mu \mathrm{eV}, \mathrm{QD} 1)$ and for a dot with a significant FSS ( $\sim 45 \mu \mathrm{eV}$, QD2), respectively. The upper panels show unpolarized spectra, $I_{\sigma}+I_{\sigma^{*}}$, where $I_{\sigma}\left(I_{\sigma^{*}}\right)$ is the emission intensity with cocircular (crosscircular) polarization with respect to the excitation light. The lower panels show the differential spectra, $I_{\sigma}-I_{\sigma^{*}}$, where a pronounced positive peak appears for the $X$ line of QD1, but disappears for that of QD2. The positive degree of polarization for the $X$ line of QD1 $(+4.2 \%)$ is evidence of the degenerate exciton states. By contrast, the $X X$ line does not exhibit a significant polarization in both QD1 and QD2, because the transition from $X X$ comprises two routes with orthogonal polarizations. The other spectral lines follow well-known dynamics: $X^{+}$shows a positive degree of polarization, which is due to spin-polarized electron injection. $X^{-}$shows a negative degree of polarization, which is accompanied by the spin-flip relaxation of electrons $[43,44]$.

Note that only a few dots exhibit circular polarization for the $X$ line. A rough estimation of the probability of finding dots with a circularly polarized $X$ line is $\sim 2 \%$, which agrees with the ratio of the natural width of our dots
(0.5 $\mu \mathrm{eV})$ divided by the distribution of FSS $(25 \mu \mathrm{eV})$. Note that this probability is smaller than that previously observed for GaAs/AlGaAs QDs on (111)A ( 5\%) [29]. The small probability of telecom-wavelength dots reflects the relatively long emission lifetime as compared with that of visible-wavelength dots (560 ps for GaAs dots). Optical orientation therefore serves as an efficient way to select dots suited for entangled photon generation.

The advantage of using (111) substrates over using (100) substrates is that we can exclude in-plane asymmetry on average. Nevertheless, shape variation inherent in the dot self-assembly gives rise to small but significant FSS in the majority of dots. Moreover, recent theoretical attempts suggest the influence of atomic-scale symmetry breaking on the emergence of FSS [45]. Such microscopic asymmetry comes from interfacial randomness at heterosurfaces and compositional fluctuations inside and outside dots. It is noteworthy that the distribution of the measured FSS in our dots is smaller than that theoretically predicted for telecom dots on (100) surfaces, in which a perfectly symmetric shape was assumed [46]. This implies that the [111] grown dots are more stable against microscopic disorder than the [100] grown dots.

We attribute the further reduction of FSS in the [111] grown dots compared with that of conventional [100] grown dots to two mechanisms. First, owing to the high surface stability of the (111) plane, the dots have atomically flat surfaces, which were demonstrated by transmission electron microscopy analysis [30]. The smooth and abrupt interface also leads to the observation of distinct spectral multiplets in ensemble spectra [Fig. 2(a)]. Thus, we expect the effect of interfacial randomness on FSS to be greatly suppressed compared with SK-grown (100) dots. Second, in zinc-blende compound semiconductors, the piezoelectric field direction is along the [111] polar axis, which coincides with the vertical growth direction in our system. Thus, a strain field does not induce any great reduction in lateral symmetry. The (111) surface is thus an ideal substrate for the growth of symmetric dots, where both geometrical (shape) symmetry and atomic-scale symmetry are well conserved.

In summary, we have presented measurements of minimized FSS in telecom-wavelength InAs QDs on an InP(111)A substrate prepared by droplet epitaxy. Polarization-resolved PL measurements were performed to examine the FSS distribution. Resolution-limited splittings (smaller than $4 \mu \mathrm{eV}$ ) were confirmed. The random distribution of the polarization axis made it possible to find symmetric dots over a wide spectral range. Thus our InAs/InAlAs dots on (111) substrates can play a crucial role in quantum information processing as an efficient entangled photon source that can work in telecom fiber networks.

This work was partly supported by Grant-in-Aid from JSPS and ERC Grant No. 306719.
[1] A. Zeilinger, Rev. Mod. Phys. 71, S288 (1999); N. Gisin, G. Ribordy, W. Tittel, and H. Zbinden, ibid. 74, 145 (2002).

[2] O. Benson, C. Santori, M. Pelton, and Y. Yamamoto, Phys. Rev. Lett. 84, 2513 (2000).
[3] K. Takemoto, Y. Nambu, T. Miyazawa, K. Wakui, S. Hirose, T. Usuki, M. Takatsu, N. Yokoyama, K. Yoshino, A. Tomita, S. Yorozu, Y. Sakuma, and Y. Arakawa, Appl. Phys. Express 3, 092802 (2010). 
[4] C. H. Bennett, G. Brassard, C. Crépeau, R. Jozsa, A. Peres, and W. K. Wootters, Phys. Rev. Lett. 70, 1895 (1993).

[5] M. Żukowski, A. Zeilinger, M. A. Horne, and A. K. Ekert, Phys. Rev. Lett. 71, 4287 (1993); S. Bose, V. Vedral, and P. L. Knight, Phys. Rev. A 57, 822 (1998); J.-W. Pan, D. Bouwmeester, H. Weinfurter, and A. Zeilinger, Phys. Rev. Lett. 80, 3891 (1998).

[6] Entanglement in time-bin basis using quantum dot cascade was also recently reported [7].

[7] H. Jayakumar, A. Predojević, T. Kauten, T. Huber, G. S. Solomon, and G. Weihs, Nat. Commun. 5, 4251 (2014).

[8] D. Gammon, E. S. Snow, B. V. Shanabrook, D. S. Katzer, and D. Park, Phys. Rev. Lett. 76, 3005 (1996).

[9] M. Bayer, A. Kuther, A. Forchel, A. Gorbunov, V. B. Timofeev, F. Schäfer, J. P. Reithmaier, T. L. Reinecke, and S. N. Walck, Phys. Rev. Lett. 82, 1748 (1999).

[10] V. D. Kulakovskii, G. Bacher, R. Weigand, T. Kümmell, A. Forchel, E. Borovitskaya, K. Leonardi, and D. Hommel, Phys. Rev. Lett. 82, 1780 (1999).

[11] R. Seguin, A. Schliwa, S. Rodt, K. Pötschke, U. W. Pohl, and D. Bimberg, Phys. Phys. Lett. 95, 257402 (2005).

[12] M. Abbarchi, C. A. Mastrandrea, T. Kuroda, T. Mano, K. Sakoda, N. Koguchi, S. Sanguinetti, A. Vinattieri, and M. Gurioli, Phys. Rev. B 78, 125321 (2008).

[13] C. Santori, D. Fattal, M. Pelton, G. S. Solomon, and Y. Yamamoto, Phys. Rev. B 66, 045308 (2002).

[14] W. Langbein, P. Borri, U. Woggon, V. Stavarache, D. Reuter, and A. D. Wieck, Phys. Rev. B 69, 161301(R) (2004); R. J. Young, R. M. Stevenson, A. J. Shields, P. Atkinson, K. Cooper, D. A. Ritchie, K. M. Groom, A. I. Tartakovskii, and M. S. Skolnick, ibid. 72, 113305 (2005).

[15] N. Akopian, N. H. Lindner, E. Poem, Y. Berlatzky, J. Avron, D. Gershoni, B. D. Gerardot, and P. M. Petroff, Phys. Rev. Lett. 96, 130501 (2006).

[16] R. M. Stevenson, R. J. Young, P. Atkinson, K. Cooper, D. A. Ritchie, and A. J. Shields, Nature (London) 439, 179 (2006); M. A. Pooley, A. J. Bennett, R. M. Stevenson, A. J. Shields, I. Farrer, and D. A. Ritchie, Phys. Rev. Appl. 1, 024002 (2014).

[17] S. Seidl, M. Kroner, A. Högele, K. Karrai, R. J. Warburton, A. Badolato, and P. M. Petroff, Appl. Phys. Lett. 88, 203113 (2006); B. D. Gerardot, S. Seidl, P. A. Dalgarno, R. J. Warburton, D. Granados, J. M. Garcia, K. Kowalik, O. Krebs, K. Karrai, A. Badolato, and P. M. Petroff, ibid. 90, 041101 (2007).

[18] A. Muller, W. Fang, J. Lawall, and G. S. Solomon, Phys. Rev. Lett. 103, 217402 (2009)

[19] A. Dousse, J. Suffczyǹski, A. Beveratos, O. Krebs, A. Lemaître, I. Sagnes, J. Bloch, P. Voisin, and P. Senellart, Nature (London) 466, 217 (2010).

[20] M. Ghali, K. Ohtani, Y. Ohno, and H. Ohno, Nat. Commun. 3, 661 (2012); M. A. Pooley, A. J. Bennett, I. Farrer, D. A. Ritchie, and A. J. Shields, Appl. Phys. Lett. 103, 031105 (2013).

[21] R. Trotta, J. S. Wildmann, E. Zallo, O. G. Schmidt, and A. Rastelli, Nano Lett. 14, 3439 (2014).

[22] Y. H. Huo, A. Rastelli, and O. G. Schmidt, Appl. Phys. Lett. 102, 152105 (2013).

[23] R. Singh and G. Bester, Phys. Rev. Lett. 103, 063601 (2009); A. Schliwa, M. Winkelnkemper, A. Lochmann, E. Stock, and D. Bimberg, Phys. Rev. B 80, 161307 (2009).

[24] Y. Sugiyama, Y. Sakuma, S. Muto, and N. Yokoyama, Appl. Phys. Lett. 67, 256 (1995); A. Hartmann, Y. Ducommun,
L. Loubies, K. Leifer, and E. Kapon, ibid. 73, 2322 (1998); L. O. Mereni, V. Dimastrodonato, R. J. Young, and E. Pelucchi, ibid. 94, 223121 (2009).

[25] E. Stock, T. Warming, I. Ostapenko, S. Rodt, A. Schliwa, J. A. Töfflinger, A. Lochmann, A. I. Toropov, S. A. Moshchenko, D. V. Dmitriev, V. A. Haisler, and D. Bimberg, Appl. Phys. Lett. 96, 093112 (2010).

[26] T. Mano, M. Abbarchi, T. Kuroda, B. McSkimming, A. Ohtake, K. Mitsuishi, and K. Sakoda, Appl. Phys. Express 3, 065203 (2010); M. Jo, T. Mano, M. Abbarchi, T. Kuroda, Y. Sakuma, and K. Sakoda, Cryst. Growth Des. 12, 1411 (2012).

[27] K. F. Karlsson, M. A. Dupertuis, D. Y. Oberli, E. Pelucchi, A. Rudra, P. O. Holtz, and E. Kapon, Phys. Rev. B 81, 161307 (2010); L. O. Mereni, O. Marquardt, G. Juska, V. Dimastrodonato, E. P. O'Reilly, and E. Pelucchi, ibid. 85, 155453 (2012)

[28] G. Juska, V. Dimastrodonato, L. O. Mereni, A. Gocalinska, and E. Pelucchi, Nat. Photonics 7, 527 (2013).

[29] T. Kuroda, T. Mano, N. Ha, H. Nakajima, H. Kumano, B. Urbaszek, M. Jo, M. Abbarchi, Y. Sakuma, K. Sakoda, I. Suemune, X. Marie, and T. Amand, Phys. Phys. B 88, 041306(R) (2013).

[30] N. Ha, X. Liu, T. Mano, T. Kuroda, K. Mitsuishi, A. Castellano, S. Sanguinetti, T. Noda, Y. Sakuma, and K. Sakoda, Appl. Phys. Lett. 104, 143106 (2014).

[31] N. I. Cade, H. Gotoh, H. Kamada, H. Nakano, and H. Okamoto, Phys. Rev. B 73, 115322 (2006).

[32] N. Chauvin, B. Salem, G. Bremond, G. Guillot, C. BruChevallier, and M. Gendry, J. Appl. Phys. 100, 073702 (2006).

[33] L. Sapienza, R. N. E. Malein, C. E. Kuklewicz, P. E. Kremer, K. Srinivasan, A. Griffiths, E. Clarke, M. Gong, R. J. Warburton, and B. D. Gerardot, Phys. Rev. B 88, 155330 (2013).

[34] M. B. Ward, M. C. Dean, R. M. Stevenson, A. J. Bennett, D. J. P. Ellis, K. Cooper, I. Farrer, C. A. Nicoll, D. A. Ritchie, and A. J. Shields, Nat. Commun. 5, 3316 (2014).

[35] N. Koguchi, S. Takahashi, and T. Chikyow, J. Cryst. Growth 111, 688 (1991); T. Mano, M. Abbarchi, T. Kuroda, C. A. Mastrandrea, A. Vinattieri, S. Sanguinetti, K. Sakoda, and M. Gurioli, Nanotechnology 20, 395601 (2009).

[36] G. Sallen, B. Urbaszek, M. M. Glazov, E. L. Ivchenko, T. Kuroda, T. Mano, S. Kunz, M. Abbarchi, K. Sakoda, D. Lagarde, A. Balocchi, X. Marie, and T. Amand, Phys. Rev. Lett. 107, 166604 (2011); G. Sallen, S. Kunz, T. Amand, L. Bouet, T. Kuroda, T. Mano, D. Paget, O. Krebs, X. Marie, K. Sakoda, and B. Urbaszek, Nat. Commun. 5, 3268 (2014).

[37] C. Zinoni, B. Alloing, C. Monat, V. Zwiller, L. H. Li, A. Fiore, L. Lunghi, A. Gerardino, H. de Riedmatten, H. Zbinden, and N. Gisin, Appl. Phys. Lett. 88, 131102 (2006); K. Takemoto, M. Takatsu, S. Hirose, N. Yokoyama, Y. Sakuma, T. Usuki, T. Miyazawa, and Y. Arakawa, J. Appl. Phys. 101, 081720 (2007); P. Miska, J. Even, O. Dehaese, and X. Marie, Appl. Phys. Lett. 92, 191103 (2008).

[38] M. Abbarchi, T. Kuroda, T. Mano, K. Sakoda, C. A. Mastrandrea, A. Vinattieri, M. Gurioli, and T. Tsuchiya, Phys. Rev. B 82, 201301(R) (2010).

[39] V. Mlinar and A. Zunger, Phys. Rev. B 80, 035328 (2009).

[40] See Supplemental Material at http://link.aps.org/supplemental/ 10.1103/PhysRevB.90.081301 for a histogram of the FSS values. 
[41] K. Kowalik, O. Krebs, A. Lemaitre, B. Eble, A. Kudelski, P. Voisin, S. Seidl, and J. A. Gaj, Appl. Phys. Lett. 91, 183104 (2007).

[42] T. Belhadj, C.-M. Simon, T. Amand, P. Renucci, B. Chatel, O. Krebs, A. Lemaître, P. Voisin, X. Marie, and B. Urbaszek, Phys. Rev. Lett. 103, 086601 (2009).

[43] S. Cortez, O. Krebs, S. Laurent, M. Senes, X. Marie, P. Voisin, R. Ferreira, G. Bastard, J.-M. Gérard, and T. Amand, Phys. Rev. Lett. 89, 207401 (2002).
[44] A. S. Bracker, E. A. Stinaff, D. Gammon, M. E. Ware, J. G. Tischler, A. Shabaev, A. L. Efros, D. Park, D. Gershoni, V. L. Korenev, and I. A. Merkulov, Phys. Rev. Lett. 94, 047402 (2005).

[45] G. Bester, S. Nair, and A. Zunger, Phys. Rev. B 67, 161306 (2003); L. He, M. Gong, C.-F. Li, G.-C. Guo, and A. Zunger, Phys. Rev. Lett. 101, 157405 (2008).

[46] E. Goldmann, S. Barthel, M. Florian, K. Schuh, and F. Jahnke, Appl. Phys. Lett. 103, 242102 (2013). 